\title{
IOT based Medical Home
}

\author{
Amrutha K. R., Haritha S. M., Haritha \\ Vasu M., Jensy A. J., Sreechithra \\ Sasidharan \\ Final year B Tech Students \\ IES College of Engineering \\ Kerala, South India
}

\author{
Jomon K. Charly \\ Assistant Professor \\ IES College of Engineering \\ Kerala, South India
}

\begin{abstract}
The technology advances in rapid pace and it contributes in the steps to facilitate the easiness of life. The real time services are emerged substantially in the world of modernization. The most important revolution of real time technology is in the field of health care scenario. It is very important to monitor various medical parameters and post operational days. Hence the latest trend in Healthcare communication method using Internet of things (IoT) is adapted. The main objective of our 'IoT based Medical home' is to continuously monitor the health status of a patient over the internet. This proposed system of health care management is specially supposed to design as a Medical Home for older patients and this is equipped such that it can be a complete protection for the older patients even when they are in lone situations. For this Real time health status updation , Real time ECG, BP, Glucose level, temperature monitoring, Automatic wheel chair access facility, Medicine reminder, Panic switch facility, Automatic ambulance calling facility are provided. This medical home is a fabulous model which has to be brought in to prevalence for the future.
\end{abstract}

\section{General Terms}

IoT. Cloud computing

\section{Keywords}

Real time health monitoring, Health care sensor networks.

\section{INTRODUCTION}

The engineering is effectively using recent advances in technology to the welfare of human kind and Nature. Recent advances in health care monitoring sensors, embedded systems and cloud computing can be used for healthcare applications. Today there are so many devices are commercially available for personal health care, and fitness[1]. This proposed system of health care management is specially supposed to design as a Medical Home for older patients and this is equipped such that it can be a complete protection for the older patients even when they are in lone situations. For this Real time health status updating , Real time ECG[3], BP, Glucose level, temperature monitoring, Automatic wheel chair access facility, Medicine reminder, Panic switch facility, Automatic ambulance calling facility are provided. A password based main entrance lock system in the home only for near ones and ambulance crew is also designed.

Our proposed Medical Home is meant to be a complete problem solver for the physical illness of the patients. Here what actually done is meticulously monitoring completely the patient from a remote or distant spot by relatives, doctors, clinical guides, neighbors as well as an ambulance driver in real time basis. The most salient part about the system is that it is capable of informing the critical conditions of the patient instantly to relatives, hospital and ambulance through a panic alarming message[3].The whole details regarding the patient's earlier health records can be utilized and verified well by the doctor directly without any queries when the patient approaches a new doctor and hospital[2]. The whole medical details of the patient are saved with in a cloud server which can be accessed any time by the clinical guides and these details are saved for ever and will be an easy source of future reference for the doctors during the diagnostic stages. Once a data is stored ,it is stored forever[4],[6]. Moreover, not only the real time monitoring, but also the earlier status reference also can be made possible by this technique. The specially designed main entrance lock of the proposed Medical home is a part of huge relevance in our idea. Suppose the patient is alone inside the home and due to abnormal health a measure, the patient falls fainted critically. The instant alarming messages regarding the condition along with images will be sent to relatives who are not near to the patients and doctor who does the consultation for the patient all the time[5]. And also an Alaric message of the situation is got sent to an ambulance control room. Then the ambulance can readily come to the spot to pick the patient to the hospital with in no time by opening the servomotor based door lock using an instant OTP. All these deeds can be watched constantly by the relatives from remote distance. .This medical home is a fabulous model which has to be brought in to prevalence for the future.

\section{LITERATURE REVIEW}

So far, there are so many works are used for remote health monitoring Network (WBAN) consisting of wearable sensors as the data acquisition unit, communication and networking and the service layer[1]. The wearable sensors placed on the body surface will collect all the current health parameters. The Sensors transmit the sensed data to a gateway server through a Bluetooth connection. The gateway server turns the data into an Observation and Measurement file and stores it on a remote server for later retrieval by clinicians through the internet[2],[5].Here by utilizing a cloud based medical data storage, a health monitoring system is presented in which medical Staff can access the stored information online through content service application. Visualization of the subject is also important in addition to the technology for data gathering, storage and access, medical data analysis. Cameras are employed near the patient for monitoring the patient through images[4].

For the purpose of improving the quality of medical care, more and more health care systems are intensely embracing the Radio Frequency Identification (RFID) technology in recent years, combined with the use of assorted hospital information systems (HIS) [1]. With the use of hybrid RFID and HIS technologies, hospital management can easily trace patients, medical materials and supplies, medications, and healthcare related inventory. By integrating RFID and mobile 
agent technologies, the healthcare system for the elderly has the potential of a revolutionary improvement in the future. The primary objective of that research is to build a convenient, safe and long - term home care environment for the elderly, under the premise of not affecting their daily routines as well as providing a complete and continuous care environment for them after leaving the hospital[6]. In the RFID based health care management schemes, all the medical records are stored in a Health ID card. When the card is swiped at a special system all the health records and medicinal intakes are saved and send to a local area network[1],[6].

\subsection{Demerits of the earlier systems}

- Real time monitoring and live streaming were not possible.

- The live health status updating of the patient at any time and from any part of the world cannot be achieved.

- The data can be send only to a Local area network.

- Encryption security is not present.

- Once the details of the patient are sent to the doctor, then it is lost. And the health details and current conditions are not stored anywhere.

- Concept of a virtual data storage space like cloud server is lacking there. And hence the monitored health status and consultation records are not able to get reused.

- The easy accessing of ambulance service automatically when the patient is at trouble is not present in most of the system.

- The previous health records are not available for future reference.

- When the patient approaches a new medical team, they can not avail the earlier health conditions and medicinal routines of the patient as prescribed before.

- Most of the systems do just patient monitoring and often not provide complete health coverage like wheelchair accessing, ensuring of life security by providing automatic door lock mechanism to pick a fainted patient to the hospital.

- The concept of a Medical home for the full health assistance of elders was not in practice in all of those systems.

- Messages sent and the data which can be accessed from the cloud are slower.

- Data logging like Pen drives or SD cards cannot be stored with the real time details in parallel.

- For ECG monitoring multiple numbers of electrodes have to use.

\section{PROPOSED TECHNOLOGY}

Cloud Processing has three distinct components: storage, analytics, and visualization[1]. The system is designed for long term storage of patient's biomedical information as well assisting health professionals with diagnostic information. Cloud based medical data storage and the upfront challenges have been extensively addressed in the literature.

Analytics that use the sensor data along with e-Health records that are becoming prevalent can help with diagnoses and prognoses for a number of health conditions and diseases[3].Additionally, Visualization is a key requirement for any such system because it is impractical to ask physicians to pore over the voluminous data or analyses from wearable sensors[4]. Visualization methods that make the data and analyses accessible to them in a readily digestible format are essential if the wearable sensors are to impact clinical practice[3],[4].

Integrating the Internet of things (IoT) paradigm into medical systems can further increase intelligence, flexibility and interoperability[6]. A device utilizing the IoT scheme is uniquely addressed and identifiable at anytime and anywhere through the Internet. IoT based devices in remote health monitoring systems are not only capable of the conventional sensing tasks but can also exchange information with each other, automatically connect to and tasks[2]. As exemplified in, such systems are able to provide services such as automatic alarm to the nearest healthcare exchange information with health institutes through the Internet, significantly simplifying set up and administration institute in the event of a critical accident for a supervised patient[5].

\subsection{Merits of the proposed system}

Real time health status updating of the lone living old people by relatives is the major advantage of the system. Real time ECG monitoring and other health monitoring is a merit of the system. Here the health status can be known from any part of the world using IoT. Automatic wheel chair access facility for older ones is a benefit from this system. Medicine reminder is available here. Live streaming is possible so that the health details can be under stood directly. Since, the IoT technology is used, the data accessing space is unlimited. Alert messages sent and datas accessed are very fast. The whole records can be logged into pen drive or SD cards fast and simultaneously .Panic switch facility is also provided. The Encryption is provided such that the details in the cloud server get maximum security. For measuring ECG instead of using a number of electrodes a single sensor is used as cost effective. Automatic ambulance calling when health is on trouble. Automatic password based door lock system only for the near ones is an exclusive merit of this Medical home. Complete protection for the old aged patients living in lone situations is its great importance.

\section{SYSTEM ARCHITECTURE}

The whole system of Medical Home is designed as two separate sections. The first part is placed near the patient for health monitoring. The second section is a wheel chair accessing system which can be used by the patient as he needs.

As it is seen in the Figure 1, here all the measured body parameters are primarily controlled by a At mega 328 microcontroller. The sensors attached to the body will sense all the physical parameters such as temperature, bottled glucose level, blood pressure, heart beat count per minute, Electro cardio gram record etc are measured with respect to the instructions executed at the At mega 328 microcontroller. The temperature sensor used here is LM35. Reflective optical glucose sensor TCRT5000, TCRT5000L series is used to sense the variations in the glucose level. The automatic blood pressure sensor which will apply the pressure at critical conditions and automatically calibrate the blood pressure values is provided.

All these sensed values are analog in type and converted to digital by using an ADC. These digital data are taken by Raspberry pi. An electronic panic switch is an single pole single through (SPST) switch is provided. When the switch is pressed, the +5 voltage is flown towards the At mega328 since a load resistor path is provided below it, which in turn energize the buzzer to ring and when the switch is un pressed the stored voltage level will be rushing into the ground 
through the resistor path. The ECG record is captured using a technology called PPG or Photo Plethysmography. In the heart beat sensor, the patient's capillary points like finger tip are pressed at a point of IR LED. As per the blood flow rate, variable amount of LED rays are reflected into a photodiode.
The photodiode will produce the current pulses. These are given to a band pass filter in which the usual frequency ranges of human ECG waves are preset. The output wave form will be a replica of human ECG waves. A counter is used to count the heartbeat count.

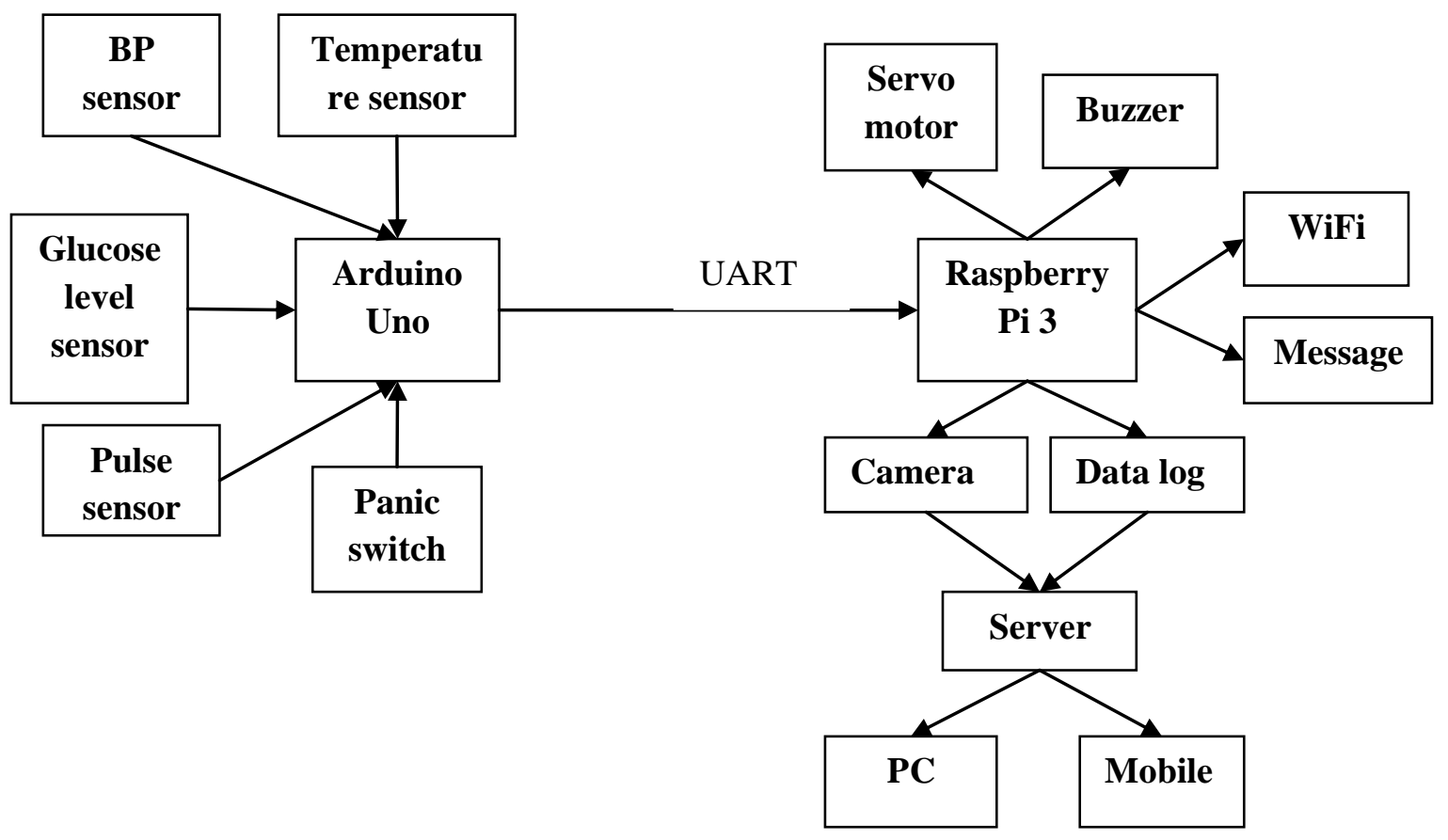

Fig 1: Block diagram of medical home system

All these measured parameters are then sent to the most advanced micro computer Raspberry Pi to carry out further processing through UART serial communication. All these datas are saved at data logging of SD card and if any measures are gone critical instant messages are sent to doctor, relatives ambulance etc and the buzzer will be rung. The real time image of the person is captured and it is also sent to the cloud server along with the other sensed details. These details can be accessed from cloud server by the relatives and family doctor through their PCs and phones by using a unique secret password with the help of Ethernet connection. For saving the fainted patient from the locked house the instantaneous password based door lock system is designed using a servomotor. This can be achieved when the password of door is sent to the mobile of ambulance crew and relatives along with the GPS location of the home. The datas saved in the cloud server are in encrypted form to ensure safety.

In the wheel chair facility(Figure 2), the WiFi connection is attached to the PIC16f877a by using the UART communication. The WiFi module is connected to the pins of PIC16F877A. The RC0 pin of PIC 16f877a is connected to the first input of motor driver L293D. RC1pin PIC16F877A is connected to $2^{\text {nd }}$ input of motor driver.RC2 and $\mathrm{RC} 3$ are connected $3^{\text {rd }}$ and $4^{\text {th }}$ pins of L293D respectively. From out 1 and out 2 of the L293D, motor1 of wheel chair is attached . From out 3 and 4 of L293D, motor 2 of wheel chair is attached. These 230RPM DC motors will act as the two wheels of the Wheel chair.

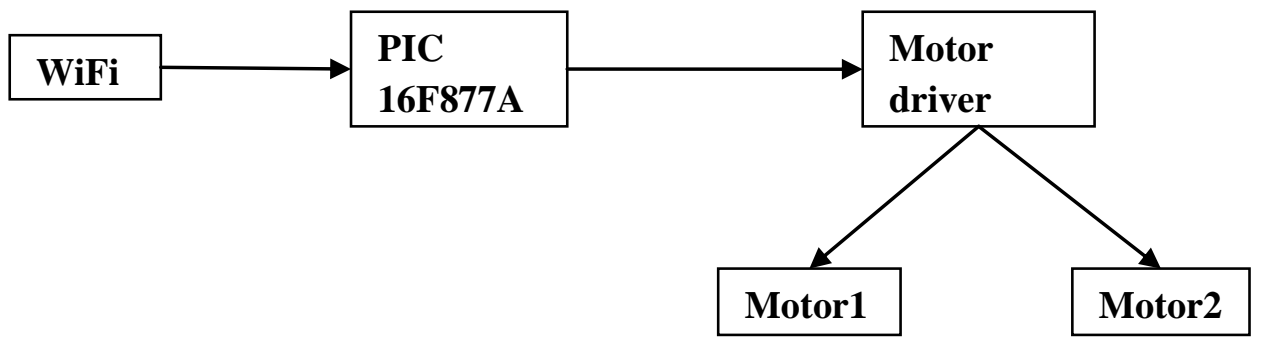

Fig 2: Block diagram of Wheel chair accessing facility

\section{COMPONENTS DETAILS}

The Arduino Uno board is used for the processing of sensed parameters and all these digitized values are sent to the RaspberryPi 3 through UART serial communication. Arduino Uno is a micro controller board based on the Atmega 328p datasheet. Raspberry Pi is an advanced mini computer with quad core ARM 7 cortex processor. The datas sent to the Raspberry Pi will be stored in the cloud storage server. Here, the server used is the 'Telegram' application. The other health care sensors used in the network are described in detail below. 


\subsection{Pulse sensor}

Pulse Sensor is a well-designed plug-and-play heart-rate sensor for Arduino. It can be used by students, artists, athletes, makers, and game \& mobile developers who want to easily incorporate live heart rate data into their projects. The sensor clips onto a fingertip or earlobe and plugs right into Arduino. It also includes an open-source monitoring app that graphs your pulse in real time. The front of the sensor is the pretty side with the Heart logo. This is the side that makes contact with the skin. On the front you see a small round hole, which is where the LED shines through from the back, and there is also a little square just under the LED. The square is an ambient light sensor, exactly like the one used in cell phones, tablets, and laptops, to adjust the screen brightness in different light conditions. The LED shines light into the fingertip or earlobe, or other capillary tissue, and sensor reads the amount of light that bounces back. The other side of the sensor is where the rest of the parts are mounted. We put them there so they would not get in the way of the of the sensor on the front. Heart beat is updated in every $2 \mathrm{~ms}$.

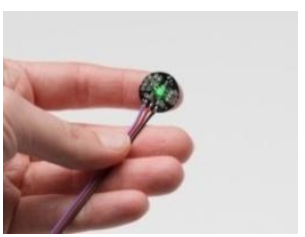

Fig 3: Pulse sensor

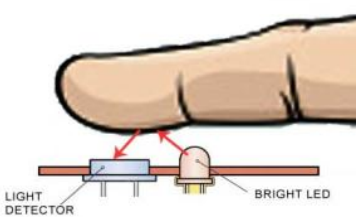

Fig 4: PPG

The current pulse output measured will be digitized via a comparator. The digital pulse outputs from comparator are fed to a Band pass filter which will confine the frequency range of this output into normal ECG frequency range of a humen being. This filter output is plotted on $\mathrm{Y}$ axis with respect to the time instants on the $\mathrm{X}$ axis by means of Java programming. Thus we can get a plot which is very closely resembled like a human ECG. This is how the ECG is established using photo plethysmography.

\subsection{Blood pressure monitoring}

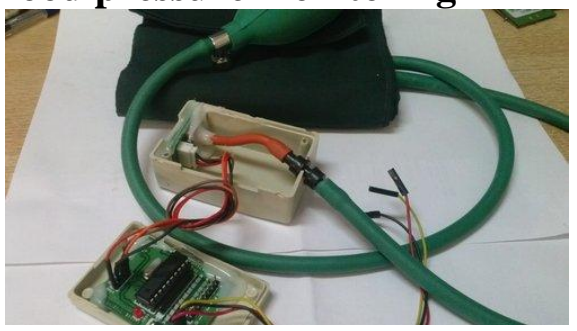

Fig 5: Sphygmomanometer

Blood pressure is the force of blood against the walls of arteries. Blood pressure is recorded as two numbers-the systolic pressure (as the heart beats) over the diastolic pressure (as the heart relaxes between beats). The measurement is written one above or before the other, with the systolic number on top and the diastolic number on the bottom. For example, a blood pressure measurement of $120 / 80 \mathrm{mmHg}$ (millimeters of mercury) is expressed verbally as "120over80".

\subsection{Glucose level monitoring}

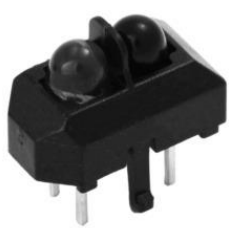

Fig 6: Glucose level sensor

The TCRT5000 and TCRT5000L are reflective sensors which include an infrared emitter and phototransistor in a leaded package which blocks visible light. The package includes two mounting clips. TCRT5000L is the long lead version. Here, as a proto type, when the arm closed near between the IR pair, ie , LED and Photo diode, the interruption made by this will reduce the ray flow in between the two. So it will lead to the indication of low glucose level in the bottle. Other wise no indications.

\subsection{Body temperature monitoring}

The LM35 series are precision integrated-circuit temperature sensors, whose output voltage is linearly proportional to the Celsius (Centigrade) temperature. The LM35 thus has an advantage over linear temperature sensors calibrated in $\S$ Kelvin, as the user is not required to subtract a large constant voltage from its output to obtain convenient Centigrade scaling. Low cost is assured by trimming and calibration at the wafer level. It can be used with single power supplies, or with plus and minus supplies.

The LM35 analog output voltage level is given to one of the 6 analog pins of arduino. Arduino has a10 bit ADC. So, $2^{10}$ or 1024 combinations are possible. That much number of voltage levels can be represented as binary digit codes. The maximum operating voltage level of LM35 is $5 \mathrm{v} . \quad(5 \mathrm{v} / 1024)=4.8 \mathrm{mv}$. $1 \mathrm{mv}=(1 / 10)^{0} \mathrm{c}$.And, when the binary value multiplied by 0.483 will produce a temperature value in degree Celsius. This out put value is termed as analog read. The LM35 can be applied easily in the same way as other integrated-circuit temperature sensors. It can be glued or cemented to a surface and its temperature will be within about $0.01 \S \mathrm{C}$ of the surface temperature. This presumes that the ambient air temperature is almost the same as the surface temperature; if the air temperature were much higher or lower than the surface temperature, the actual temperature of the LM35 die would be at an intermediate temperature between the surface temperature and the air temperature. This is especially true for the TO-92 plastic package, where the copper leads are the principal thermal path to carry heat into the device, so its temperature might be closer to the air temperature than to the surface temperature. To minimize this problem, be sure that the wiring to the LM35, as it leaves the device, is held at the same temperature as the surface of interest. The easiest way to do this is to cover up these wires with a bead of epoxy which will insure that the leads and wires are all at the same temperature as the surface, and that the LM35 die's temperature will not be affected by the air temperature. 


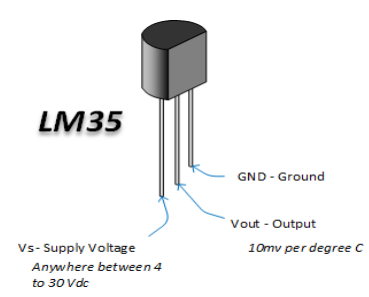

Fig 7: Temperature sensor

\section{SOFTWARE DESIGN}

\subsection{The program is uploaded in Raspberry Pi using the following steps}

- Double click the icon python IDLE.

- A new window named Python 27.13 shell is appeared.

- $\quad$ Take 'File'. And click 'New'.

- A new untitled window is appeared.

- Write the programme on the window.

- Go to Run option and then auto run

- Go to Run module F5 and save the programme.

- Now, the results and error are displayed in python 2.7 shell.

\subsection{The program is burnt in the Arduino Uno using the following steps}

- Connect the Arduino using the USB cable.

- Choose Tools $\rightarrow$ Board $\rightarrow$ Arduino Uno to find the board in the Arduino menu.

- $\quad$ Choose the correct serial port for your board.

- Click the Upload button.

\subsection{Algorithms for the programming sections are as follows}

\subsubsection{Algorithm for IoT section}

Step 1: Start

Step 2: Initialize WiFi connection

Step 3: Checking for internet connection

Step 4: If connection is ok, go to step 5, else go to step 3.

Step 5: Update application programme interface.

Step 6: Waiting for Authorization. If Ok, go to step 7.

Step 7: Waiting for commands.

Step 8: If commands come, go to it's job, else pass.

Step 9: End

\subsubsection{Working Algorithm for processing the} datas in Arduino uno.

\section{Step1: Start}

Step 2: Initialize UART communication

Step 3: Initialize analog to digital conversion and communication.
Step 4: Read ADC channels

Step 5: Send ADC values serial.

Step 6: End

\subsubsection{Algorithm to control Wheel chair using Android application}

Step 1: Start

Step 2: Initialize the UART communication.

Step 3: Initialize WiFi

Step 4: Checking WiFi connection, if yes go to step5, else go to step 3 .

Step 5: Setting data direction registers.

Step 6: If UART data $==$ ' $u$ ' set port $0 \mathrm{~b} 00000101$

Step 7: If UART data=='d' set port 0b00001010

Step 8: If UART data $==$ ' 1 ' set port $0 \mathrm{~b} 00000001$

Step 9: If UART data =='r' set port $0 \mathrm{~b} 00000100$

Step 10: End

\subsubsection{Algorithm to plot ECG from PPG values.}

Step1: Start

Step 2: Initialize UART communication

Step 3: Read UART values

Step 4: Plot serial communication values.

Step 5: End

\section{HARDWARE DESIGN}

\subsection{PCB layout}

The PCB layout is a mirrored positive one - black on white. Mirrored as viewed from the silkscreen top (component) side. The PCB layout is printed 1:1 on paper by means of a laser printer or copier machine. The laser printer or copier toner will not run out when it gets wet or oily. The ink of an inkjet paper print does run out and inkjet printers are therefore useless with the described method.

\subsection{PCB Etching}

The developed PCB is etched with a $220 \mathrm{~g} / \mathrm{l}$ solution of ammonium peroxy disulfate (NH4)2S2O8, ie, ammonium persulfate, 220 gram added to 1 liter of water and mix it until everything is dissolved. Theoretically it should be possible to etch slightly more than 60 grams of copper with 1 liter etching solution. Assume an 50\% efficiency, about 30 grams of copper. With a thickness of $35 \mu \mathrm{m}$ copper on your PCB this covers a copper area of about $1000 \mathrm{~cm} 2$. Unfortunately the efficiency of the etching solution degrades, dissolved ammonium peroxydisulfate decomposes slowly. It is better to make just enough etching solution to etch. For an etching tray of about $20 \times 25 \mathrm{~cm}$ a minimum practical amount is $200-250$ $\mathrm{ml}$ solution. So dissolve about 44 grams ammonium peroxydisulfate into $200 \mathrm{ml}$ or 55 grams into $250 \mathrm{ml}$ water.

Etching at ambient temperature might take over an hour, it is better to heat up the etching solvent to about 35-45 degrees Celcius. The etching solution heating up could be done in a magnetron, this takes about 40 to 60 seconds in a $850 \mathrm{~W}$ 
magnetron depending on the initial temperature of the etching solution. The etching - rocking the etching tray takes about 15-30 minutes at this, if we have a heated air-bubble circulated etching fluid tank available, this is probably the fastest way to etch. At higher temperatures the etching performance decreases. The etching process is an exothermic reaction, it generates heat. Cooling the etching tray is necessary. When starting the etching process and little to etch it is difficult to keep the etching solution at 35-45 degrees Celcius. It helps to fill for example the kitchen sink with warm water and rock the etching tray in the filled kitchen sink. When the ammonium peroxydisulfate is dissolved it is a clear liquid. After an etching procedure it gradually becomes blue and more deeper blue Compared to other etching chemicals like hydrated iron chloride $\mathrm{FeCl} 3.6 \mathrm{H} 2 \mathrm{O}$ ie, ferric chloride or the combination of hydrochloric acid HCL and hydrogen peroxide $\mathrm{H}_{2} \mathrm{O}_{2}$, using ammonium peroxy di sulfate is a clean and safe method. However, copper sulfate is a poisonous substance and should be treated as chemical waste. Here the etching process and PCB fabrication are done for the Wheel chair accessing arrangement.

\subsection{Prototype of system}

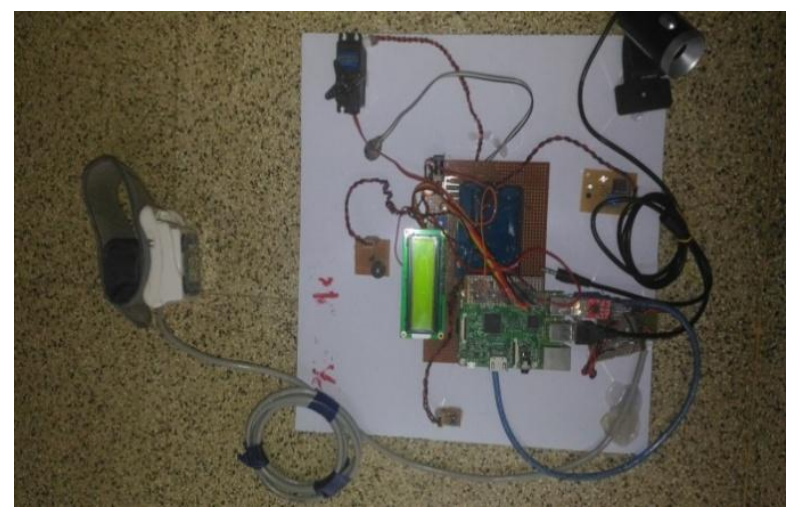

Fig 8: Patient monitoring Section

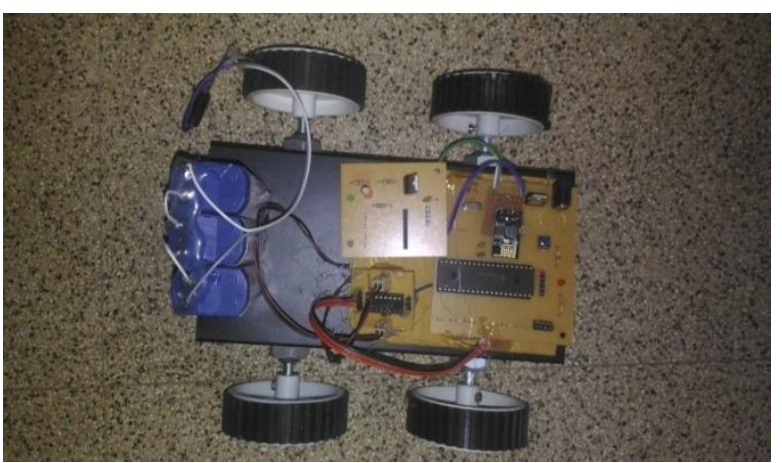

Fig 9: Wheel Chair Section

We use the app 'Telegram' as server and we can control the system by sending the commands on the app. We use the app 'Telnet' for WiFi connection.

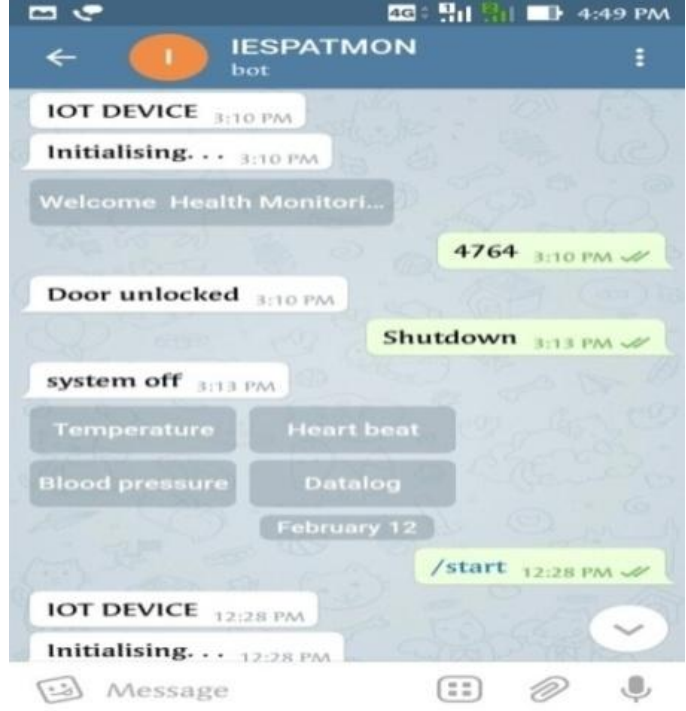

Fig 10: Display of Patient Health Details in Telegram

\section{CASE STUDY AND PROBLEMS FACED}

As we tried to create an Advanced medical home appliance, we had to collaborate different technical systems such as Android controlled wheel chair, Automatic OTP based door lock system etc together. We had to use four different programming languages such as c, embedded c, Java, Python to implemented the Medical Home system. We first planned to implement the wheel chair system as a real model. But, in practice, we could make it as a proto type only. We made a four wheeled locomotive model controlled by two dc motors. We could not prepare a real model of door lock system . Instead, we have just shown the rotation of the servomotor according to OTP entered in Telegram. Since we combined multiple systems together to increase the facilities the cost of implementation was high.

\section{CONCLUSION}

In this paper, we reviewed the methods to provide complete healthcare aids for a patient who is in lack of near ones with him. Wearable sensors collect the body parameters and store them in a cloud space. These datas are accessed via internet. Wheel chair facility and password based door lock system contributes the more convenient life for patients who are living in the home itself. Learning of new programming languages such as Python, Embedded C, Java etc was a new experience for us. After completing the project, we feel so proud and contented that we could contribute a very useful initiative for the innovations in the medical field and we are so satisfied with our efforts to complete this project successfully.

\section{ACKNOWLEDGMENT}

The authors would like to acknowledge the Management, Principal, Head of the department, all the faculties especially our project guide Mr. Jomon K.Charly and all staff of Electronics and Communication Department, of IES College of Engineering, Thrissur, Kerala, for their aid and technical guidance given during the entire course to complete it providentially. Also we extend our sincere thanks to the entire $\mathrm{R} \& \mathrm{D}$ team of the college for giving the facility to do our project in the R \& D lab. 


\section{REFERENCES}

[1] Moeen Hassanalieragh, Alex Page, Tolga Soyata, G Sharma Mehmet Aktas, and Gonzalo Mateos, "Health Monitoring Using Internets of-Things (IoT) Sensing with Cloud-based and Management Processing: Opportunities and the Challenges", 2015 IEEE Conference on Services Computing International

[2] A. Pantelopoulos and N. Bourbakis "A survey on wearable sensor- based on The systems for health monitoring and prognosis", IEEE Trans. Sys., Man, and the Cybernetics,Part C: Applic. and wq Reviews, vol. 40, no.1, pp.1-12, Jan 2010

[3] A. Page, O. Kocabas, T. Soyata, M. Aktas, and J.P.Couderc, "Cloud- Based On PAN Privacy-Preserving Remote ECG Monitoring and Surveillance", Annalsis of The Noninvasive Electrocardiology (ANEC), 2014
[4] R. Paradiso, G.Loriga, and N. Taccini, "A wearable health care System Based on knitted integrated sensors" ,IEEE Trans. Info. Tech. in Biomedicine, vol. 9, no. 3, pp. 337-344, in Biomedicine, vol. 9, no. 3, pp. 337344, Sept 2005.

[5] A. Milenkovi, C. Otto, and E. Jovanov, "Wireless sensor networks for the Persons", The Noninvasive Electrocardiology (ANEC), 2014.

[6] M. Bazzani, D. Conzon, A. Scalera, M. Spirito, and C. Trainito, "Enabling the IoT paradigm in e-health solutions through the VIRTUS middleware",in IEEE 11th Int. Conf. n Trust, Security and Privacy in Computing and Com,June 2012, pp. 1954-1959. 